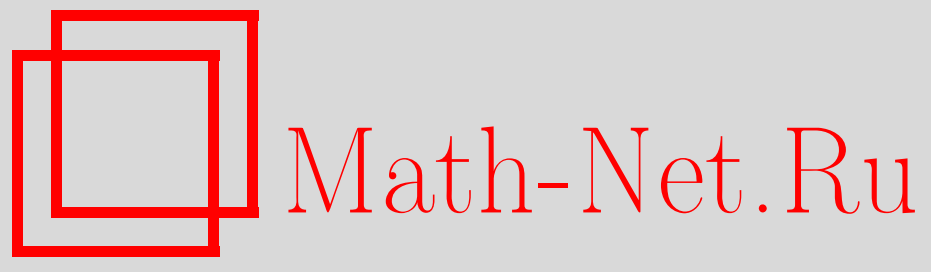

Т. Г. Хессами Пилеруд, Х. Хессами Пилеруд, Об одной гипотезе Эрдеша, Матем. заметки, 2008, том 83, выпуск $2,312-315$

DOI: https://doi.org/10.4213/mzm4422

Использование Общероссийского математического портала Math-Net.Ru подразумевает, что вы прочитали и согласны с пользовательским соглашением http://www . mathnet.ru/rus/agreement

Параметры загрузки:

IP: 3.85 .5 .30

26 апреля 2023 г., 14:55:30

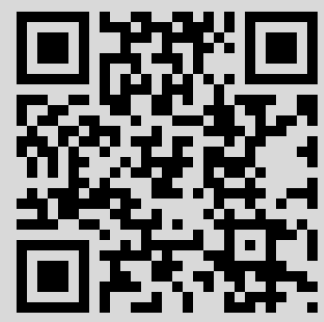




\section{Об одной гипотезе Эрдеша}

\section{Т. Хессами Пилеруд, Х. Хессами Пилеруд}

В работе будут использоваться следующие стандартные обозначения: $\mathbb{Z}$ - кольцо целых чисел, $\mathbb{Q}$ и $\overline{\mathbb{Q}}$ - поля рациональных и алгебраических чисел соответственно, $\varphi(q)$ функция Эйлера, $\omega(q)$ - количество различных простых делителей числа $q$.

В 1960-х гг. Эрдеш высказал следующее предположение [1; с. 430], которое до сих пор не доказано и не опровергнуто: для любого натурального $q$ и произвольной числовой функиии $f(n)$ с периодом $q$, принимающей значения $+1,-1$ при $n=1,2, \ldots, q-1$ и 0 при $n=q$, сумма сходящегося ряда

$$
S=\sum_{n=1}^{\infty} \frac{f(n)}{n}
$$

отлична от нуля. Заметим, что необходимым и достаточным условием сходимости ряда является равенство

влекущее нечетность $q$.

$$
\sum_{n=1}^{q} f(n)=\sum_{n=1}^{q-1} f(n)=0,
$$

В 1969 г. Чоула поставил вопрос о существовании периодической функции $g: \mathbb{Z} \rightarrow \mathbb{Q}$ с простым периодом $q$, для которой

$$
\sum_{n=1}^{\infty} \frac{g(n)}{n}=0 .
$$

Отрицательный ответ на этот и даже более общий вопрос был дан в работе Бейкера, Берча и Вирзинга [2]. Ими было доказано, что если $q$-периодическая, не равная тождественно нулю функция $g: \mathbb{Z} \rightarrow \overline{\mathbb{Q}}$ удовлетворяет следующим условиям:

1) $g(r)=0$ при $1<(r, q)<q$;

2 ) если круговой многочлен $\Phi_{q}$ неприводим над $\mathbb{Q}(g(1), \ldots, g(q))$, то сумма сходящегося ряда $\sum_{n=1}^{\infty} g(n) / n$ отлична от нуля.

В 1982 г. Окада [3], используя [2], сформулировал теорему, описывающую все $q$-периодические функции $g: \mathbb{Z} \rightarrow \overline{\mathbb{Q}}$, для которых $\sum_{n=1}^{\infty} g(n) / n=0$, при условии, что круговой многочлен $\Phi_{q}$ неприводим над $\mathbb{Q}(g(1), \ldots, g(q))$. Указанным критерием явилась система $\varphi(q)+\omega(q)$ однородных линейных уравнений с рациональными коэффициентами от неизвестных $g(1), \ldots, g(q)$. С помощью этого критерия в [3] удалось дать частичный положительный ответ на предположение Эрдеша, заключающийся в том, что сумма $S \neq 0$, если $2 \varphi(q) \geqslant q$, т.е. для всех тех натуральных $q$, для которых $\omega(q) \leqslant 2$. Используя работу [3], Тайдеман [4] доказал справедливость гипотезы Эрдеша для всех мультипликативных функций $f$. Результат Окады был улучшен Сарадой в [5], показавшей, что $S \neq 0$, если $2 \varphi(q) \geqslant q(1-1 / h)$, где $h=\max _{1 \leqslant i \leqslant k}\left(p_{i}^{t_{i}}\right)$ и $q=p_{1}^{t_{1}} \cdots p_{k}^{t_{k}}$. В той же работе был исследован вопрос о справедливости предположения Эрдеша при $\omega(q)=3$ и 4.

Заметим, что если $g-q$-периодическая функция, то сумма сходящегося ряда

$$
\sum_{n=1}^{\infty} g(n) / n
$$

Работа выполнена при поддержке Института исследований по теоретической физике и математике (гранты №№ 86110020 (первый автор) и 86110025 (второй автор).

(C) Т. ХесСами Пилеруд, Х. ХЕСсами ПилЕрУд, 2008 
может быть представлена в виде

$$
\begin{aligned}
\sum_{n=1}^{\infty} \frac{g(n)}{n} & =\sum_{k=0}^{\infty} \sum_{p=1}^{q} \frac{g(p)}{q k+p}=\sum_{k=0}^{\infty} \sum_{p=2}^{q} g(p)\left(\frac{1}{k q+p}-\frac{1}{k q+1}\right) \\
& =\frac{1}{q} \sum_{p=2}^{q} g(p)\left(\psi\left(\frac{1}{q}\right)-\psi\left(\frac{p}{q}\right)\right)=\frac{-1}{q} \sum_{p=1}^{q} g(p) \psi\left(\frac{p}{q}\right)
\end{aligned}
$$

где

$$
\psi(z)=\frac{d}{d z} \log \Gamma(z)=-\gamma+\sum_{n=0}^{\infty}\left(\frac{1}{n+1}-\frac{1}{n+z}\right)
$$

является логарифмической производной гамма-функции, а $\gamma=-\psi(1)$ - константа Эйлера. Из представления (1) легко следует, что предположение Эрдеша равносильно тому, что любая линейная комбинация вида

$$
\sum_{p=1}^{q-1}( \pm 1) \psi\left(\frac{p}{q}\right), \quad \text { где } q-\text { нечетное число, }
$$

отлична от нуля. В противоположность этому при четном $q$ существование аналогичной линейной комбинации

$$
\sum_{p=1}^{36}( \pm 1) \psi\left(\frac{p}{36}\right)=0
$$

было недавно доказано Тенгели [6]. Более точно, им была доказана следующая теорема.

Теорема 1. Существует функиия $f: \mathbb{N} \rightarrow\{-1,1\}$ с периодом 36 , для которой

$$
\sum_{n=1}^{\infty} \frac{f(n)}{n}=0
$$

Доказательство Тенгели основано на обширном компьютерном переборе и предъявлении функции $f(n)=1,-1,-1,-1,-1,1,1,1,-1,1,-1,-1,1,-1,1,-1,-1,1,1,1,-1,1,-1$, $-1,1,-1,-1,-1,-1,1,1,1,1,1,-1,1$ при $n=1,2, \ldots, 36$, которая удовлетворяет критерию Окады [3; теорема 10]. Кроме того, им было показано, что 36 - это минимальный период функции $f$, указанной в теореме.

Мы дадим здесь другое элементарное доказательство теоремы 1, основанное на следующем функциональном соотношении для логарифмической производной гамма-функции $[7 ; \S 1.7 .1]$ :

$$
\psi(m z)=\frac{1}{m} \sum_{r=0}^{m-1} \psi\left(z+\frac{r}{m}\right)+\log m .
$$

Доказательство теоремы 1. Рассмотрим соотношение (2) при $m=6$ :

$$
6 \psi(6 z)=\sum_{r=0}^{5} \psi\left(z+\frac{r}{6}\right)+6 \log 6 .
$$

Беря разность двух тождеств, полученных из (3) при подстановке $z=1 / 36$ и $z=5 / 36$, имеем

$$
\sum_{r=0}^{5}\left(\psi\left(\frac{1+6 r}{36}\right)-\psi\left(\frac{5+6 r}{36}\right)\right)=6 \psi\left(\frac{1}{6}\right)-6 \psi\left(\frac{5}{6}\right) .
$$


Рассматривая соотношение (2) при $m=3$ и подставляя последовательно $z=1 / 18$ и $z=5 / 18$, получим

$$
3 \psi\left(\frac{1}{6}\right)-3 \psi\left(\frac{5}{6}\right)=\sum_{r=0}^{2}\left(\psi\left(\frac{1+6 r}{18}\right)-\psi\left(\frac{5+6 r}{18}\right)\right) .
$$

Аналогично из (2) при $m=2, z=1 / 12$ и $z=5 / 12$ находим

$$
2 \psi\left(\frac{1}{6}\right)-2 \psi\left(\frac{5}{6}\right)=\sum_{r=0}^{1}\left(\psi\left(\frac{1+6 r}{12}\right)-\psi\left(\frac{5+6 r}{12}\right)\right) .
$$

Окончательно из (4)-(6) получаем

$$
\begin{aligned}
\sum_{r=0}^{5}( & \left.\psi\left(\frac{1+6 r}{36}\right)-\psi\left(\frac{5+6 r}{36}\right)\right) \\
= & \sum_{r=0}^{2}\left(\psi\left(\frac{1+6 r}{18}\right)-\psi\left(\frac{5+6 r}{18}\right)\right) \\
& +\sum_{r=0}^{1}\left(\psi\left(\frac{1+6 r}{12}\right)-\psi\left(\frac{5+6 r}{12}\right)\right)+\psi\left(\frac{1}{6}\right)-\psi\left(\frac{5}{6}\right) .
\end{aligned}
$$

Далее из (2) при $m=2, z=1 / 6$ и $z=1 / 3$ находим

$$
3 \psi\left(\frac{1}{3}\right)-3 \psi\left(\frac{2}{3}\right)=\psi\left(\frac{1}{6}\right)-\psi\left(\frac{5}{6}\right) .
$$

С другой стороны, подставляя в (2) $m=3, z=1 / 9$ и $z=2 / 9$, имеем

$$
3 \psi\left(\frac{1}{3}\right)-3 \psi\left(\frac{2}{3}\right)=\sum_{r=0}^{2}\left(\psi\left(\frac{1+3 r}{9}\right)-\psi\left(\frac{2+3 r}{9}\right)\right) .
$$

Следовательно, с учетом (8), (9) мы можем переписать (7) в виде

$$
\begin{aligned}
\sum_{r=0}^{5}( & \left.\psi\left(\frac{1+6 r}{36}\right)-\psi\left(\frac{5+6 r}{36}\right)\right)+\sum_{r=0}^{2}\left(\psi\left(\frac{5+6 r}{18}\right)-\psi\left(\frac{1+6 r}{18}\right)\right) \\
& +\sum_{r=0}^{1}\left(\psi\left(\frac{5+6 r}{12}\right)-\psi\left(\frac{1+6 r}{12}\right)\right)+\sum_{r=0}^{2}\left(\psi\left(\frac{2+3 r}{9}\right)-\psi\left(\frac{1+3 r}{9}\right)\right)=0 .
\end{aligned}
$$

Используя нетривиальную обнуляющуюся линейную комбинацию вида

$$
\psi(1)-\psi\left(\frac{1}{3}\right)-\psi\left(\frac{2}{3}\right)-\psi\left(\frac{1}{4}\right)-\psi\left(\frac{3}{4}\right)+\psi\left(\frac{1}{2}\right)+\psi\left(\frac{1}{6}\right)+\psi\left(\frac{5}{6}\right)=0,
$$

которая может быть получена из (2) при выборе $m=2, z=1 / 4, z=1 / 2$ и $m=3, z=1 / 3$, $z=1 / 6$, и прибавляя ее к (10), мы получим требуемое тождество

$$
\begin{aligned}
\sum_{r=0}^{5}( & \left.\psi\left(\frac{1+6 r}{36}\right)-\psi\left(\frac{5+6 r}{36}\right)\right)+\sum_{r=0}^{2}\left(\psi\left(\frac{5+6 r}{18}\right)-\psi\left(\frac{1+6 r}{18}\right)\right) \\
& +\sum_{r=0}^{1}\left(\psi\left(\frac{5+6 r}{12}\right)-\psi\left(\frac{1+6 r}{12}\right)\right)+\sum_{r=0}^{2}\left(\psi\left(\frac{2+3 r}{9}\right)-\psi\left(\frac{1+3 r}{9}\right)\right) \\
& +\psi\left(\frac{1}{6}\right)+\psi\left(\frac{5}{6}\right)-\psi\left(\frac{1}{4}\right)-\psi\left(\frac{3}{4}\right)-\psi\left(\frac{1}{3}\right)-\psi\left(\frac{2}{3}\right)+\psi\left(\frac{1}{2}\right)+\psi(1)=0
\end{aligned}
$$


В заключение отметим, что все рассмотренные в настоящей заметке бесконечные ненулевые суммы являются трансцендентными числами, как было показано в [8; теорема 1], что также легко следует из представления (1), формулы Гаусса для логарифмической производной гамма-функции $[9 ; \S 1.2$, с. 14$]$

$$
\psi\left(\frac{p}{q}\right)=-\gamma-\log q+\sum_{k=1}^{q-1} \omega^{-k p} \log \left(1-\omega^{k}\right), \quad \text { где } \quad p, q \in \mathbb{Z}, \quad 0<p<q, \quad \omega=e^{2 \pi i / q},
$$

и теории Бейкера о линейных формах от логарифмов алгебраических чисел.

\section{СПИСОК ЦИТИРОВАННОЙ ЛИТЕРАТУРЫ}

[1] A.E. Livingston, Canad. Math. Bull., 8 (1965), 413-432. [2] A. Baker, B. J. Birch, E. A. Wirsing, J. Number Theory, 5:3 (1973), 224-236. [3] T. Okada, Acta Arith., 40:2 (1982), 143-153. [4] R. Tijdeman, Number Theory for the Millennium, III, A K Peters, Natick, MA, 2002, 261-284. [5] N. Saradha, The Riemann Zeta Function and Related Themes, Proceedings of the conference in honour of K. Ramachandra, 2, Ramanujan Math. Soc., 2006, 121-129; http://www.math.tifr.res.in/ saradha/papers.html. [6] R. Tijdeman, More Sets, Graphs and Numbers, Bolyai Soc. Math. Stud., 15, Springer, Berlin, 2006, 381-405; http://www.math.leidenuniv.nl/ ${ }^{2}$ ijdeman/tijbud.ps. [7] A. Erdelyi, W. Magnus, F. Oberhettinger, G. F. Tricomi, Higher Transcendental Functions, Vol. 1. Based, in part, on notes left by Harry Bateman, McGraw-Hill, New York, 1953. [8] S. D. Adhikari, N. Saradha, T. N. Shorey, R. Tijdeman, Indag. Math. (N.S.), 12:1 (2001), 1-14. [9] G. E. Andrews, R. Askey, R. Roy, Special Functions, Encyclopedia Math. Appl., 71, Cambridge Univ. Press, Cambridge, 1999.

\section{Т. Хессами Пилеруд}

Shahrekord University, Iran,

Institute for Studies in Theoretical

Physics and Mathematics (IPM), Iran

E-mail: hessamit@ipm.ir

\section{Х. Хессами Пилеруд}

Shahrekord University,

Institute for Studies in Theoretical

Physics and Mathematics (IPM)

E-mail: hessamik@ipm.ir 